\title{
Article
}

\section{Application Multi-Fuzzy Soft Sets in Hypermodules}

\author{
Osman Kazanci ${ }^{1,+}+\mathbb{D}$, Sarka Hoskova-Mayerova ${ }^{2, *,+}+\mathbb{D}$ and Bijan Davvaz ${ }^{3,+}$ (D) \\ 1 Department of Mathematics, Karadeniz Technical University, Trabzon 61080, Turkey; okazanci@ktu.edu.tr \\ 2 Department of Mathematics and Physics, University of Defence, Kounicova 65, 66210 Brno, Czech Republic \\ 3 Department of Mathematics, Yazd University, Yazd 89136, Iran; davvaz@yazd.ac.ir \\ * Correspondence: sarka.mayerova@unob.cz; Tel.: +420-973-44-2225 \\ + These authors contributed equally to this work.
}

check for

updates

Citation: Kazanc1, O.;

Hoskova-Mayerova, S.; Davvaz, B. Application Multi-Fuzzy Soft Sets in Hypermodules. Mathematics 2021, 9, 2182. https://doi.org/10.3390/ math9182182

Academic Editor: Michael Voskoglou

Received: 6 July 2021

Accepted: 1 September 2021

Published: 7 September 2021

Publisher's Note: MDPI stays neutral with regard to jurisdictional claims in published maps and institutional affiliations.

Copyright: (c) 2021 by the authors. Licensee MDPI, Basel, Switzerland. This article is an open access article distributed under the terms and conditions of the Creative Commons Attribution (CC BY) license (https:/ / creativecommons.org/licenses/by/ $4.0 /)$.

\begin{abstract}
The goal of this paper is to introduce a novel soft hyperstructure called multi-fuzzy soft hyperstructure. We investigate the notion of multi-fuzzy soft hypermodules and some of their structural properties are discussed. We discuss the behavior image and inverse image of a multi-fuzzy soft set under the multi-fuzzy soft function. According to Zadeh's extension principle, we prove that the image and inverse image of a multi-fuzzy soft hypermodules are further multi-fuzzy soft hypermodule.
\end{abstract}

Keywords: multi-fuzzy set; multi-fuzzy soft set; multi-fuzzy soft hypermodule

MSC: 20N20; 20N25; 08A72

\section{Introduction}

Algebraic hyperstructures based on hyperoperations were introduced by Marty [1] and studied by many scientists. There are several books on hyperstructure theory, see in [2-5]. In [2], many applications are mentioned [2]. For example, fuzzy and rough set theory, cryptography, codes,automata, probability, geometry, lattices, binary relations, graphs, and hypergraphs. Studying fuzzy hyperalgebraic structures is one of the important topics of fuzzy set theory. Fuzzy sets, defined by Zadeh [6], and hyperstructures, defined by Marty [1], have found wide application area both theoretically and practically. The relationship between fuzzy sets and algebraic hyperstructures has been considered by some researchers. We recommend to readers to see the works in [7-12]. In [8,9], Davvaz applied the notion of fuzzy sets to the theory of algebraic hyperstructures and defined fuzzy subhypergroup (resp. $H_{v}$-subgroup) of a hypergroup (resp. $H_{v}$-group), which is a generalization of the notion of Rosenfeld's fuzzy subgroup of a group [10] and also defined fuzzy $H_{v}$-submodules. Different hypermodules, fuzzy hypermodules, and rough hypermodules have been studied [13-19].

Soft set theory introduced by Molodtsov [20] is an powerful mathematical tool for modeling vagueness. After Molodsov's work, some different applications of soft sets were studied in [21,22]. Maji et al. [22] offered the definition of fuzzy soft set. They presented the first practical application of soft sets in decision making problems. The more generalized concept of the fuzzy soft set, which is a combination of fuzzy sets and soft sets, introduced and studied by these authors. Both soft set and fuzzy soft set theories have been applied to algebraic structures and hyperstructures by some authors, see in [23-27] for the relation between the generalization of crisp sets.

Recently, the theory of multi-fuzzy set has been introduced by Sebastian and Ramakrishnan [28]. Multi-fuzzy set theory uses ordered sequences of membership function and provides a new method to represent problems unexplained in other extensions of fuzzy set theory. In [29], Onasanya and Hoskova-Mayerova introduced fuzzy multi-groups excited by multisets. Then, fuzzy multi-polygroups and fuzzy multi- $H_{v}$-ideals, which are a generalization of fuzzy $H_{v}$-ideals, were studied in [30,31]. Yang et al. [32] proposed the 
concept of the multi-fuzzy soft set for its application to decision making, which is a more general fuzzy soft set.

The notion of multi-fuzzy soft sets provides a new method to represent some problems, which are difficult to explain in other extensions of fuzzy set theory. For example, in a twodimensional image, the color of pixels cannot be characterized by a membership function of an ordinary fuzzy set, but it can be characterized by a three-dimensional multi-fuzzy soft sets. Figure 1 shows the relation between generalization of crisp sets. Lately, Akın [33] proposed the multi-fuzzy soft sets to the theory of groups and defined the multi-fuzzy soft group.

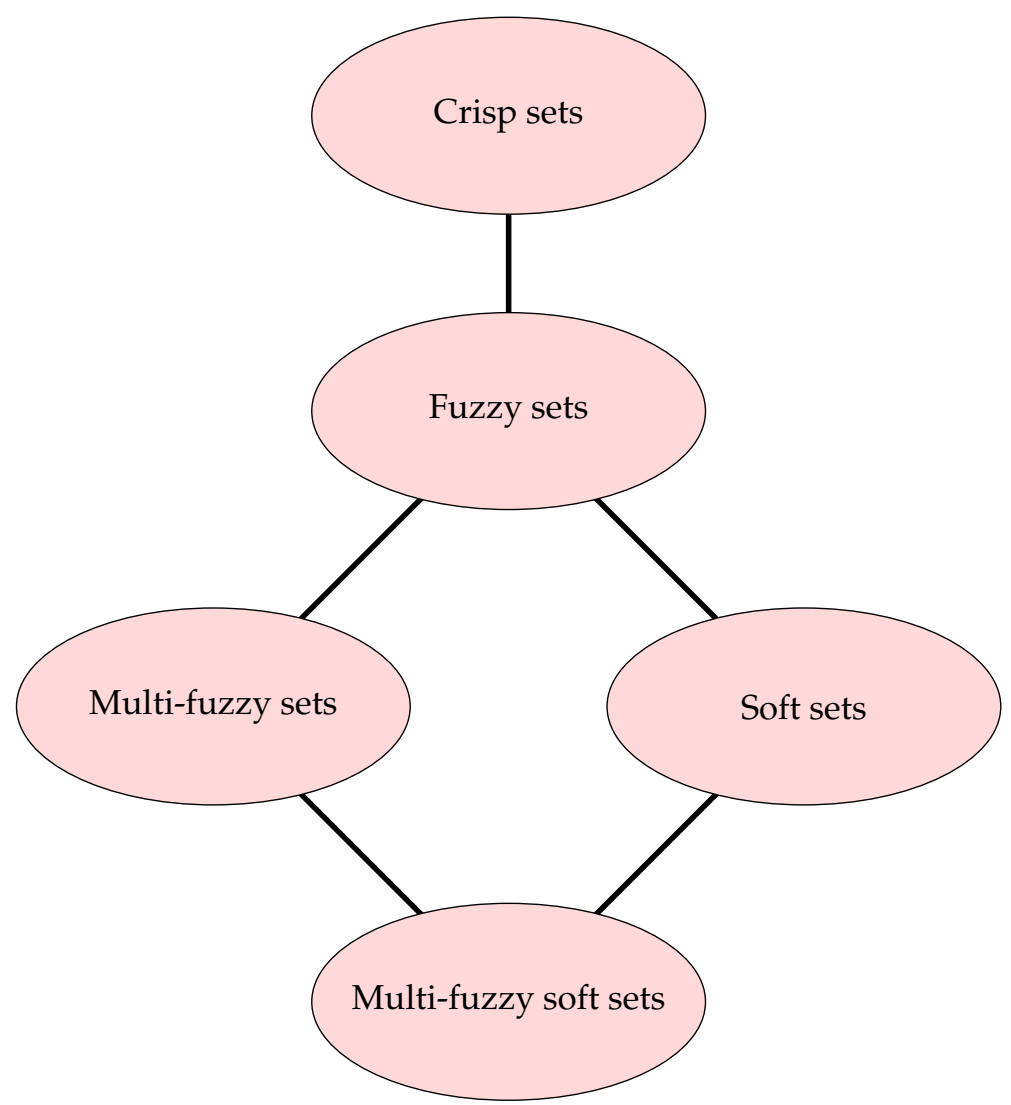

Figure 1. The relation between generalization of crisp sets.

The goal of this paper is to assemble fuzzy soft hypermodules and soft hypermodules, from which we obtain a new sort of soft hypermodule named multi-fuzzy soft hypermodule. We first recall some basic definitions and results which are used to present the article in Section 2. In Section 3, the notion of multi-fuzzy soft hypermodule with its properties and structural characterization are discussed. In Section 4, we give the image and inverse image of a multi-fuzzy soft set under the multi-fuzzy soft function, and then define soft homomorphism between multi-fuzzy soft hypermodules. In Section 5, some conclusions are presented.

\section{Preliminaries}

Let $H$ be a basic non-empty set. A hyperoperation on $H$ is a mapping $\circ: H \times H \rightarrow$ $\mathcal{P}^{*}(H)$, where $\mathcal{P}^{*}(H)$ is the set of all the non-empty subsets of $H$. If $x \in H$ and $A, B \in$ $\mathcal{P}^{*}(H)$, then we denote $A \circ B, A \circ x$, and $x \circ B$ as follows: $A \circ B=\bigcup_{a \in A, b \in B} a \circ b, A \circ x=$ $A \circ\{x\}$ and $x \circ B=\{x\} \circ B .(H, \circ)$ is called a canonical hypergroup [5] if the following axioms hold:

(1) $x \circ(y \circ z)=(x \circ y) \circ z$, for all $x, y, z \in H$;

(2) $x \circ y=y \circ x$, for all $x, y, z \in H$; 
(3) There exists $e \in H$ such that $e \circ x=x$, for all $x \in H$;

(4) for every $x \in H$ there exists a unique element $x^{\prime} \in H$ such that $e \in x \circ x^{\prime},\left(x^{\prime}\right.$ is called the adverse of $x$ ).

Definition 1 (Refs. [34-36]). The algebraic structure $(R,+, \cdot)$ is called a hyperring (Krasner hyperring) if the following axioms hold: $(1)(R,+)$ is a canonical hypergroup; (we shall write 0 and $-x$ for e and $x^{\prime}$, respectively); (2) $(R, \cdot)$ is a semigroup having zero as a bilaterally absorbing element; (3) The multiplication is distributive with respect to the hyperoperation + .

It is easy to see that he following elementary facts follow from the axioms: $-(-x)=x$ and $-(x+y)=-x-y$. Let $A$ be a non-empty subset of a hyperring $R$. Then $A$ is called a sub-hyperring of $R$ if $(A,+, \cdot)$ is itself a hyperring. A sub-hyperring $A$ of $R$ is called a normal if $x+A-x \subseteq A$ for all $x \in R$.

Definition 2 (Ref. [16]). Let $M$ be a non-empty set. Then, $M$ is called a hypermodule over a hyperring $R$ ( $R$-hypermodule) if $(M,+)$ is a canonical hypergroup and there exists a map $\cdot: R \times M \rightarrow \mathcal{P}^{*}(M)$ by $(r, m) \mapsto r m$ such that for all $r_{1}, r_{2} \in R$ and $m_{1}, m_{2} \in M$, we have $r_{1}\left(m_{1}+m_{2}\right)=r_{1} m_{1}+r_{2} m_{2},\left(r_{1}+r_{2}\right) m_{1}=r_{1} m_{1}+r_{2} m_{1}$, and $\left(r_{1} r_{2}\right) m_{1}=r_{1}\left(r_{2} m_{1}\right)$.

Let $A$ be a non-empty subset of hypermodule $M$. Then, $A$ is called a sub-hypermodule of $M$ if $A$ is itself a hypermodule. A sub-hypermodule $A$ of $M$ is called a normal if $x+A-x \subseteq A$ for all $x \in M$. From the definition, the following propositions are obtained. Let $A$ be a normal sub-hypermodule of $M$. Then, $(A+x)+(A+y)=A+x+y$ for all $x, y \in M$ and $A+x=A+y$ for all $y \in A+x$. If $A$ and $B$ are sub-hypermodules of an $R$-hypermodule $M$ with $B$ normal in $M$, then $A \cap B$ is a normal sub-hypermodule of $A$ and $B$ is a normal sub-hypermodule of $A+B$. A mapping $f: M \rightarrow M^{\prime}$ is called a homomorphism if for all $a, b \in M$ and $r \in R, f(a+b)=f(a)+f(b), f(r a)=r f(a)$ and $f(0)=0$. Clearly, a homomorphism $f$ is an isomorphism if $f$ is both injective and surjective.

Definition 3 (Ref. [11]). Let $\mu$ be a fuzzy subset of a hypermodule M over a R. $\mu$ is called a fuzzy sub-hypermodule of $M$ if

(1) $\min \{\mu(x), \mu(y)\} \leq \inf _{\alpha \in x+y}\{\mu(\alpha)\}$ for all $x, y \in M$;

(2) $\mu(x) \leq \mu(-x)$ for all $x \in M$;

(3) $\mu(x) \leq \mu(r x)$ for all $r \in R$ and $x \in M$.

A fuzzy sub-hypermodule $\mu$ of $M$ is called a normal if $\mu(y) \leq \inf _{\alpha \in x+y-x} \mu(\alpha)$ for all $x, y \in M$. Let $\mu$ be a fuzzy sub-hypermodule of $M$. It is clear that $\mu(-x)=\mu(x)$, $\min \{\mu(x), \mu(y)\} \leq \inf _{z \in x-y}\{\mu(z)\}$, for all $x, y \in M$.

We first recall some basic definitions and results which are used to present the article $[20,23-26,28,32]$. Let $U$ be an initial universe set and $E$ be a set of parameters. $\mathcal{P}(U)$ denotes the power set of $U$ and $A \subseteq E$.

Definition 4 (Ref. [20]). $(F, A)$ is said to be a soft set over $U$, where $F$ is a set-valued function $F: A \rightarrow \mathcal{P}(U)$ can be defined as $F(x)=\{y \in U \mid(x, y) \in R\}$ for all $x \in A$ and $R$ will refer to an arbitrary binary relation between an element of $A$ and an element of $U$, that is, $R$ is a subset of $A \times U$. Actually, a soft set over $U$ is a parameterized family of subsets of the universe $U$.

Definition 5 (Ref. [22,24]). Let $F S(U)$ be all fuzzy subsets of $U$. Then, $(f, A)$ is said to be a fuzzy soft set over $U$, where $f$ is a mapping given by $f: A \rightarrow F S(U)$. That is, for each $a \in A$, $f(a)=f_{a}: U \rightarrow[0,1]$ is a fuzzy set on $U$.

Definition 6 (Ref. [28]). A multi-fuzzy set $\widetilde{A}$ in $U$ is a set of ordered sequences $\widetilde{A}=\left\{u /\left(\mu_{1}(u), \mu_{2}(u), \ldots, \mu_{k}(u)\right\}\right.$, where $\mu_{i} \in F S(U) i=1,2, \ldots, k$ and $k$ is a positive integer. 
The function $\mu_{\widetilde{A}}=\left(\left(\mu_{1}(u), \mu_{2}(u), \ldots, \mu_{k}(u)\right)\right.$ is called the multi membership function of multi-fuzzy set $\widetilde{A}$ denoted by $M M_{\widetilde{A}}, k$ is called a dimension of $\widetilde{A}$. Denoted by $M^{k} F S(U)$, the set of all multi-fuzzy sets of dimension $k$ in $U$.

Note: Zadeh's fuzzy set is one-dimensional multi-fuzzy set and Atanassov's intuotionistic fuzzy set is two-dimensional multi-fuzzy set with $\mu_{1}(u)+\mu_{2}(u) \leq 1$

Example 1 (Ref. [28]). Assume a color image is approximated by an mxn matrix of pixels. Let $U$ be the set of all pixels of the color image. For any pixel $u \in U$, the membership values $\mu_{r}(u), \mu_{g}(u)$, $\mu_{b}(u)$ are the normalized red value, green value and blue value of the pixel $u$, respectively. Therefore, the color image can be estimated by the collection of pixels with the multi-membership function $\left(\mu_{r}(u), \mu_{g}(u), \mu_{b}(u)\right)$ and can be represented as a multi-fuzzy set $\widetilde{A}=\left\{u /\left(\mu_{r}(u), \mu_{g}(u), \mu_{b}(u) \mid\right.\right.$ $u \in U\}$. In a two-dimensional image, pixel color cannot be characterized by a membership function of an ordinary fuzzy set. However, pixel color can be qualified by a three-dimensional membership function $\left(\mu_{r}(u), \mu_{g}(u), \mu_{b}(u)\right)$. Actually, a multi-fuzzy set can be known as a more general fuzzy set using ordinary fuzzy sets as its building blocks.

Definition 7 (Ref. [28]). Let $\widetilde{A} \in M^{k} F S(U)$. If $\widetilde{A}=\{u /(0,0, \ldots, 0): u \in U\}$, then $\widetilde{A}$ is said to be the null multi-fuzzy set of dimension $k$, denoted by $\widetilde{\Phi}_{k}$. If $\widetilde{A}=\{u /(1,1, \ldots, 1): u \in U\}$, then $\widetilde{A}$ is said to be the absolute multi-fuzzy set of dimension $k$, denoted by $\widetilde{1_{k}}$.

Definition 8 (Ref. [28]). Let $\widetilde{A}=\left\{u /\left(\mu_{1}(u), \mu_{2}(u), \ldots, \mu_{k}(u)\right): u \in U\right\}$ and $\widetilde{B}=\left\{u /\left(v_{1}(u)\right.\right.$, $\left.\left.v_{2}(u), \ldots, v_{k}(u)\right): u \in U\right\}$ be two multi-fuzzy sets of dimension $k$ in $U$. We give the following relations and operations.

(1) $\widetilde{A} \sqsubseteq \widetilde{B}$ if and only if $M M_{\widetilde{A}} \leq M M_{\widetilde{B}}$, i.e., $\mu_{i}(u) \leq v_{i}(u), \forall u \in U$ and $1 \leq i \leq k$.

(2) $\widetilde{A}=\widetilde{B}$ if and only if $M M_{\widetilde{A}}=M M_{\widetilde{B}}$, i.e., $\mu_{i}(u)=v_{i}(u), \forall u \in U$ and $1 \leq i \leq k$.

(3) $\widetilde{A} \sqcup \widetilde{B}=\left\{u /\left(\mu_{1}(u) \vee v_{1}(u), \mu_{2}(u) \vee v_{2}(u), \ldots, \mu_{k}(u) \vee v_{k}(u)\right): u \in U\right\}$. That is, $M M_{\widetilde{A} \sqcup \widetilde{B}}=M M_{\widetilde{A}} \vee M M_{\widetilde{B}}$

(4) $\widetilde{A} \sqcap \widetilde{B}=\left\{u /\left(\mu_{1}(u) \wedge v_{1}(u), \mu_{2}(u) \wedge v_{2}(u), \ldots, \mu_{k}(u) \wedge v_{k}(u)\right): u \in U\right\}$. That is, $M M_{\widetilde{A} \sqcap \widetilde{B}}=M M_{\widetilde{A}} \wedge M M_{\widetilde{B}}$.

(5) $\widetilde{A^{c}}=\left\{u /\left(\mu_{1}^{c}(u), \mu_{2}^{c}(u), \ldots, \mu_{k}^{c}(u): u \in U\right\}\right.$

Definition 9 (Ref. [32]). A pair $(\widetilde{f}, A)$ is called a multi-fuzzy soft set of dimension $k$ over $U$, where $\widetilde{f}$ is a function defined by $\widetilde{f}: A \rightarrow M^{k} F S(U)$. That is, for each $a \in A, \widetilde{f}(a)=M M_{\widetilde{f}(a)} \in$ $M^{k} F S(U)$. For $a \in A, \widetilde{f}(a)$ may be considered a set of a-approximate elements of the multi-fuzzy $\operatorname{soft} \operatorname{set}(\widetilde{f}, A)$.

Definition 10 (Ref. [32]). Let $(\widetilde{f}, A)$ and $(\widetilde{g}, B)$ be two multi-fuzzy soft sets of dimension $k$ over $U$ where $A, B \subseteq E$. Then, $(\widetilde{f}, A)$ is said to be a multi-fuzzy soft subset of $(\widetilde{g}, B)$ if, $A \subseteq B$ and for each $a \in A \widetilde{f}(a) \sqsubseteq \widetilde{g}(a)$. In this situation, we write $(\widetilde{f}, A) \widetilde{\Xi}(\widetilde{g}, B)$.

Definition 11 (Ref. [32]). Let $(\widetilde{f}, A)$ be a multi-fuzzy soft set of dimension $k$ over $U$. Then, $(\widetilde{f}, A)$ is called a null multi-fuzzy soft set, denoted by $\widetilde{\Phi_{A}^{k}}$ if $\widetilde{f}(a)=\widetilde{\Phi_{k}}$ for all $a \in A$.

$(\widetilde{f}, A)$ is called a absolute multi-fuzzy soft set, denoted by $\widetilde{U_{A}^{k}}$ if $\widetilde{f}(a)=\widetilde{1_{k}}$ for all $a \in A$.

Definition 12 (Ref. [32]). Let $(\widetilde{f}, A)$ and $(\widetilde{g}, B)$ be two multi-fuzzy soft sets of dimension $k$ over U.

(1) Their $\wedge$-intersection denoted by $(\widetilde{f}, A) \widetilde{\wedge}(\widetilde{g}, B)$, is defined as $(\widetilde{f}, A) \widetilde{\wedge}(\widetilde{g}, B)=(\widetilde{h}, A \times B)$, where $\widetilde{h}(a, b)=\widetilde{f}(a) \sqcap \widetilde{g}(b)$, for all $(a, b) \in A \times B$.

(2) Their $\vee$-union denoted by $(\widetilde{f}, A) \widetilde{V}(\widetilde{g}, B)$, is defined as $(\widetilde{f}, A) \widetilde{V}(\widetilde{g}, B)=(\widetilde{h}, A \times B)$, where $\widetilde{h}(a, b)=\widetilde{f}(a) \sqcup \widetilde{g}(b)$, for all $(a, b) \in A \times B$. 
Definition 13 (Ref. [32]). Let $(\widetilde{f}, A)$ and $(\widetilde{g}, B)$ be two multi-fuzzy soft sets of dimension $k$ over $U$. Then, their union is the multi-fuzzy soft set $(\widetilde{h}, C)$, where $C=A \cup B$ and for all $c \in C$, $\widetilde{h}(c)=\widetilde{f}(c)$ if $c \in A-B, \widetilde{h}(c)=\widetilde{g}(c)$ if $c \in B-A$ and $\widetilde{h}(c)=\widetilde{f}(c) \sqcup \widetilde{g}(c)$ if $c \in A \cap B$. We write $(\widetilde{f}, A) \widetilde{\sqcup}(\widetilde{g}, B)=(\widetilde{h}, C)$.

Definition 14 (Ref. [32]). Let $(\widetilde{f}, A)$ and $(\widetilde{g}, B)$ be two multi-fuzzy soft sets of dimension $k$ over $U$.

(1) Their restricted intersection is the multi-fuzzy soft set $(\widetilde{h}, C)$, where $C=A \cap B \neq \varnothing$, and for all $c \in C, \widetilde{h}(c)=\widetilde{f}(c) \sqcap \widetilde{g}(c)$. Its denoted by $(\widetilde{f}, A) \widetilde{\Pi}_{\Re}(\widetilde{g}, B)=(\widetilde{h}, C)$.

(2) Their extended intersection is the multi-fuzzy soft set $(\hat{h}, C)$, where $C=A \cup B$ and for all $c \in C, \widetilde{h}(c)=\widetilde{f}(c)$ if $c \in A-B, \widetilde{h}(c)=\widetilde{g}(c)$ if $c \in B-A$ and $\widetilde{h}(c)=\widetilde{f}(c) \sqcap \widetilde{g}(c)$ if $c \in A \cap B$. We write $(\widetilde{f}, A) \widetilde{\Pi}_{\Im}(\widetilde{g}, B)=(\widetilde{h}, \stackrel{C}{ })$.

\section{Multi-Fuzzy Soft Hypermodules}

In [30], the notion of fuzzy-multi polygroup which extends the notion of multi-fuzzy group studied by Al Tahan et all. In this paper, we used the definition algebraic structure of multi-fuzzy set and multi-fuzzy soft set which was given by Sebastian et.al in [28] and Yang et al. [32], respectively, we introduce the definition of multi-fuzzy soft hypermodules and give some fundamental properties of multi-fuzzy soft hypermodules.

Definition 15. Let $M$ be a hypermodule over a hyperring $R$ and $(\widetilde{f}, A)$ be a multi-fuzzy soft set of dimension $k$ over $M$. Then $(\tilde{f}, A)$ is called a multi-fuzzy soft hypermodule of dimension $k$ over $M$ if and only if for all $a \in A, x, y \in M$ and $r \in R$,

(1) $\min \left\{M M_{\widetilde{f}(a)}(x), M M_{\widetilde{f}(a)}(y)\right\} \leq \inf _{z \in x+y}\left\{M M_{\widetilde{f}(a)}(z)\right\}$;

(2) $M M_{\widetilde{f}(a)}(x) \leq M M_{\widetilde{f}(a)}(-x)$;

(3) $M M_{\widetilde{f}(a)}(x) \leq M M_{\widetilde{f}(a)}(r x)$.

That is, for each $a \in A, \widetilde{f}(a)=M M_{\widetilde{f}(a)}$ is a multi-fuzzy subhypermodule of $M$.

The first condition requires that the hypermodule is closed under multi-fuzzy soft hyperoperation + . The second condition is a generalization of inverse element under + . Furthermore, the third condition extend the external product.

Proposition 1. Let $(\widetilde{f}, A)$ be a multi-fuzzy soft set of dimension $k$ over $M$. If $(\widetilde{f}, A)$ is a multifuzzy soft hypermodule, then for all $x, y \in M$ and $a \in A$, we have

(i) $\min \left\{M M_{\widetilde{f}(a)}(x), M M_{\widetilde{f}(a)}(y)\right\} \leq \inf _{z \in x-y}\left\{M M_{\widetilde{f}(a)}(z)\right\}$,

(ii) $M M_{\widetilde{f}(a)}(x)=M M_{\widetilde{f}(a)}(-x)$,

(iii) $M M_{\widetilde{f}(a)}(x) \leq M M_{\widetilde{f}(a)}(0)$.

Proof. The proof follows from Definition 15.

Indeed, the condition (i) is equivalent to the conditions (1) and (2). The condition (ii) means that the degree of membership of $-x$ is equal to $x$, and the condition (iii) says that the degree of 0 is greater than of all elements of $M$.

To explain, we give a few examples to see what the definition means.

Example 2. Consider $M=\{0,1,2,3\}$ and $R=\{0,1,2\}$ with the following hyperoperations:

\begin{tabular}{c|cccc}
+1 & 0 & 1 & 2 & 3 \\
\hline 0 & 0 & 1 & 2 & 3 \\
1 & 1 & 0 & $\{1,2\}$ & $\{1,3\}$ \\
2 & 2 & $\{1,2\}$ & 0 & $\{2,3\}$ \\
3 & 3 & $\{1,3\}$ & $\{2,3\}$ & 0
\end{tabular}

\begin{tabular}{l|llll}
$\cdot 1$ & 0 & 1 & 2 & 3 \\
\hline 0 & 0 & 0 & 0 & 0 \\
1 & 0 & 1 & 2 & 3 \\
2 & 0 & 2 & 0 & 2
\end{tabular}




\begin{tabular}{c|ccc}
+2 & 0 & 1 & 2 \\
\hline 0 & 0 & 1 & 2 \\
1 & 1 & 0 & $\{1,2\}$ \\
2 & 2 & $\{1,2\}$ & 0
\end{tabular}

\begin{tabular}{l|lll}
$\cdot 2$ & 0 & 1 & 2 \\
\hline 0 & 0 & 0 & 0 \\
1 & 0 & 1 & 2 \\
2 & 0 & 2 & 1
\end{tabular}

Then, $\left(M,+_{1}, \cdot_{1}\right)$ is a hypermodule over hyperring $\left(R,+_{2}, \cdot 2\right)$ [26]. Let $A=\left\{e_{1}, e_{2}, e_{3}\right\}$ be the set of parameters.

(1) We define the multi-fuzzy soft set $\tilde{f}: A \rightarrow M^{3} F S(M)$ as follows.

$$
\begin{aligned}
& M M_{\widetilde{f}\left(e_{1}\right)}=\{0 /(0.8,0.7,0.6), 1 /(0.6,0.5,0.4), 2 /(0.7,0.6,0.5), 3 /(0.2,0.2,0.4)\} \\
& M M_{\widetilde{f}\left(e_{2}\right)}=\{0 /(0.6,0.7,0.8), 1 /(0.5,0.5,0.4), 2 /(0.6,0.6,0.8), 3 /(0.3,0.3,0.2)\} \\
& M M_{\widetilde{f}\left(e_{3}\right)}=\{0 /(0.9,0.9,0.8), 1 /(0.2,0.2,0.4), 2 /(0.6,0.4,0.6), 3 /(0.5,0.2,0.4)\}
\end{aligned}
$$

Then, for all $a \in A, M M_{\widetilde{f}(a)}$ is a multi-fuzzy subhypermodule on M. By Definition 15, $(\widetilde{f}, A)$ is a multi-fuzzy soft hypermodule of dimension 3 over $M$.

(2) We define the multi-fuzzy soft set $\widetilde{f}: A \rightarrow M^{3} F S(M)$ as follows.

$$
\begin{aligned}
M M_{\widetilde{f}\left(e_{1}\right)} & =\{0 /(0.6,0.6,0.7), 1 /(0.4,0.5,0.4), 2 /(0.5,0.5,0.6), 3 /(0.4,0.2,0.3)\} \\
M M_{\widetilde{f}} & =\{0 /(0.9,0.9,0.7), 1 /(0.6,0.4,0.2), 2 /(0.7,0.5,0.4), 3 /(0.8,0.6,0.5)\} \\
M M_{\widetilde{f}\left(e_{3}\right)} & =\{0 /(0.6,0.7,0.7), 1 /(0.3,0.2,0.4), 2 /(0.5,0.5,0.6), 3 /(0.2,0.2,0.3)\}
\end{aligned}
$$

Then, it is easy to see that $M M_{\widetilde{f}\left(e_{1}\right)}$ and $M M_{\widetilde{f}\left(e_{3}\right)}$ are multi-fuzzy subhypermodules on $M$. However, $M M_{\widetilde{f}\left(e_{2}\right)}$ is not a multi-fuzzy subhypermodule on $M$ as $\left.M M_{\widetilde{f}\left(e_{2}\right)}(2.3)\right\}=M M_{\widetilde{f}\left(e_{2}\right)}(2) \nsupseteq$ $M M_{\widetilde{f}\left(e_{2}\right)}$ (3). By Definition 15, $(\widetilde{f}, A)$ is not a multi-fuzzy soft hypermodule of dimension 3 over $M$.

Example 3. Let $A \subset E$ and $(F, A)$ be a soft set over $M$. For all $a \in A$, the multi-fuzzy soft set $\tilde{\chi}_{F(a)}: A \rightarrow M^{k} F S(M)$ defined by

$$
M M_{\widetilde{\chi}_{F(a)}}(b)=\left\{\begin{array}{cc}
\widetilde{1}_{k} & \text { if } b \in F(a) \\
\widetilde{\Phi}_{k}, & \text { otherwise }
\end{array}\right.
$$

for all $b \in A$. Then, $\left(\tilde{\chi}_{F(a)}, A\right)$ is a multi-fuzzy soft set of dimension $k$ over $M$. According to this way a soft set $(F, A)$ over $M$ can be consider as a multi-fuzzy soft set of dimension $k$ over $M$.

Example 4. According to Example 2, each one soft set can be seen as a multi-fuzzy soft set and each one characteristic function of a subhypermodule of a hypermodule is a multi-fuzzy subhypermodule, we can take into account a soft hypermodule as a multi-fuzzy soft hypermodule.

Example 5. Take into account the hypermodule $M=\{0,1,2,3\}$ defined in Example 2. Let $\varnothing \neq A$ be a set of parameters. We define the multi-fuzzy soft set $\widetilde{f}: A \rightarrow M^{k} F S(M)$ as follows. For all $a \in A M M_{\widetilde{f}(a)}(0) \geq M M_{\widetilde{f}(a)}(x)$ for all $x \in M, M M_{\widetilde{f}(a)}(1) \geq M M_{\tilde{f}(a)}(3)$ and $M M_{\widetilde{f}(a)}(2) \geq M M_{\widetilde{f}(a)}(1)$. Then, $M M_{\widetilde{f}(a)}$ is a multi-fuzzy sub-hypermodule of $M$ for all $a \in A$. By Definition 15, $(\widetilde{f}, A)$ is a multi-fuzzy soft hypermodule of dimension $k$ over $M$.

The following theorem shows that Definition 15 is a generalization of soft hypermodule. The theorem is expressed in terms of a necessary and sufficient condition.

Theorem 1. Let $F: A \rightarrow \mathcal{P}^{*}(P)$ be a soft set over $M$. Then, $(F, A)$ is a soft hypermodule over $M$ if and only if $\left(\widetilde{\chi}_{F(a)}, A\right)$ is a multi-fuzzy soft hypermodule of dimension $k$ over $M$.

Proof. The proof is follows by Example 3. 
In Theorem 2, we show that the restricted intersection and the extended intersection of two multi-fuzzy soft hypermodules are also multi-fuzzy soft hypermodules.

Theorem 2. Let $(\widetilde{f}, A)$ and $(\widetilde{g}, B)$ be two multi-fuzzy soft hypermodules of dimension $k$ over $M$. (1) $(\widetilde{f}, A) \widetilde{\Pi}_{\Re}(\widetilde{g}, B)$ is a multi-fuzzy soft hypermodule.

(2) $(\widetilde{f}, A) \widetilde{\Pi}_{\Im}(\widetilde{g}, B)$ is a multi-fuzzy soft hypermodule.

Proof. (1) Using Definition 14 (i), let $(\widetilde{f}, A) \widetilde{\Pi}_{\Re}(\widetilde{g}, B)=(\widetilde{h}, C)$, where $C=A \cap B$ and for all $c \in C, \widetilde{h}(c)=\widetilde{f}(c) \sqcap \widetilde{g}(c)$. As $(\widetilde{f}, A)$ and $(\widetilde{g}, B)$ be two multi-fuzzy soft hypermodule of dimension $k$ over $M$, we have for arbitrary $c \in C$, for all $x, y \in M$ and $r \in R$

$$
\begin{aligned}
\inf _{z \in x+y}\left\{M M_{\tilde{f}(c)}(z)\right\} & \geq \min \left\{M M_{\tilde{f}(c)}(x), M M_{\widetilde{f}(c)}(y)\right\}, \\
M M_{\tilde{f}(c)}(x) & \leq M M_{\widetilde{f}(c)}(-x) \text { and } M M_{\tilde{f}(c)}(r x) \geq M M_{\tilde{f}(c)}(x) \\
\inf _{z \in x+y}\left\{M M_{\widetilde{g}(c)}(z)\right\} & \geq \min \left\{M M_{\widetilde{g}(c)}(x), M M_{\widetilde{g}(c)}(y)\right\}, \\
M M_{\widetilde{g}(c)}(x) & \leq M M_{\widetilde{g}(c)}(-x) \text { and } M M_{\widetilde{g}(c)}(r x) \geq M M_{\widetilde{g}(c)}(x) .
\end{aligned}
$$

For arbitrary $c \in C$, for all $x, y \in M$ and $r \in R$

$$
\begin{aligned}
\inf _{z \in x+y}\left\{M M_{\widetilde{h}(c)}(z)\right\} & =\inf _{z \in x+y}\left\{M M_{\widetilde{f}(c) \sqcap \widetilde{g}(c)}(z)\right\} \\
& =\inf _{z \in x+y}\left\{M M_{\widetilde{f}(c)}(z) \wedge M M_{\widetilde{g}(c)}(z)\right\} \\
& =\inf _{z \in x+y}\left\{M M_{\widetilde{f}(c)}(z)\right\} \wedge \inf _{z \in x+y}\left\{M M_{\widetilde{g}(c)}(z)\right\} \\
& \geq \min \left\{M M_{\widetilde{f}(c)}(x), M M_{\widetilde{f}(c)}(y)\right\} \wedge \min \left\{M M_{\widetilde{g}(c)}(x), M M_{\widetilde{g}(c)}(y)\right\} \\
& =\min \left\{M M_{\widetilde{f}(c)}(x), M M_{\widetilde{g}(c)}(x)\right\} \wedge \min \left\{M M_{\widetilde{f}(c)}(y), M M_{\widetilde{g}(c)}(y)\right\} \\
& =\min \left\{M M_{\widetilde{f}(c) \sqcap \widetilde{g}(c)}(x), M M_{\widetilde{f}(c) \sqcap \widetilde{g}(c)}(y)\right\} \\
& =\min \left\{M M_{\widetilde{h}(c)}(x), M M_{\widetilde{h}(c)}(y)\right\} .
\end{aligned}
$$

Moreover,

$$
\begin{aligned}
M M_{\widetilde{h}(c)}(x) & =M M_{\widetilde{f}(c) \sqcap \widetilde{g}(c)}(x) \\
& =\min \left\{M M_{\widetilde{f}(c)}(x), M M_{\widetilde{g}(c)}(x)\right\} \\
& \leq \min \left\{M M_{\widetilde{f}(c)}(-x), M M_{\widetilde{g}(c)}(-x)\right\} \\
& =M M_{\widetilde{f}(c) \sqcap \widetilde{g}(c)}(-x) \\
& \left.=M M_{\widetilde{h}(c)}(-x) \cdot\right\} \\
M M_{\widetilde{h}(c)}(r x) & =M M_{\widetilde{f}(c) \sqcap \widetilde{g}(c)}(r x) \\
& =\min \left\{M M_{\widetilde{f}(c)}(r x), M M_{\widetilde{g}(c)}(r x)\right\} \\
& \geq \min \left\{M M_{\widetilde{f}(c)}(x), M M_{\widetilde{g}(c)}(x)\right\} \\
& =M M_{\widetilde{f}(c) \sqcap \widetilde{g}(c)}(x) \\
& =M M_{\widetilde{h}(c)}(x) .
\end{aligned}
$$

Therefore, $(\widetilde{f}, A) \widetilde{\sqcap}_{\Re}(\widetilde{g}, B)=(\widetilde{h}, C)$ is a multi-fuzzy soft hypermodule of dimension $k$ over $M$.

(2) According to Definition 14 (2), we can write $(\widetilde{f}, A) \sqcap_{\Im}(\widetilde{g}, B)=(\widetilde{h}, C), C=A \cup B$. If $c \in A-B$, then $\widetilde{h}(c)=M M_{\widetilde{h}(c)}=M M_{\widetilde{f}(c)}=\widetilde{f}(c)$ is a multi fuzzy sub-hypermodule of $M$, as $(\widetilde{f}, A)$ is a multi-fuzzy soft hypermodule over $M$; if $c \in B-A$, then $\widetilde{h}(c)=$ $M M_{\widetilde{h}(c)}=M M_{\widetilde{g}(c)}=\widetilde{g}(c)$ is a multi fuzzy sub-hypermodule of $M$, as $(\widetilde{g}, B)$ is a multi- 
fuzzy soft hypermodule over $M$; if $c \in A \cap B$, then $\widetilde{h}(c)=\widetilde{f}(c) \sqcap \widetilde{g}(c)$ is a multi-fuzzy sub-hypermodule of $M$ by (1). Therefore, $(\widetilde{f}, A) \widetilde{\Pi}_{\Im}(\widetilde{g}, B)$ is a multi-fuzzy soft hypermodule of dimension $k$ over $M$.

From Theorem 2, we have the following corollary.

Corollary 1. Let $\left\{\left(\widetilde{f}_{i}, A_{i}\right) \mid i \in I\right\}$ be a family of multi-fuzzy soft hypermodules of dimension $k$ over $M$. If $\cap_{i \in I} A_{i} \neq \varnothing$. Then,

(1) $\left(\widetilde{\Pi}_{\Re}\right)_{i \in I}\left(\widetilde{f}_{i}, A_{i}\right)$ is a multi-fuzzy soft hypermodule over $M$.

(2) $\left(\widetilde{\Pi}_{\Im}\right)_{i \in I}\left(\widetilde{f}_{i}, A_{i}\right)$ is a multi-fuzzy soft hypermodule over $M$.

The union of two multi-fuzzy soft hypermodules is not a multi-fuzzy soft hypermodule. In Theorem 3, we provide a condition for the union to be a multi-fuzzy soft hypermodule too.

Theorem 3. Let $(\widetilde{f}, A)$ and $(\widetilde{g}, B)$ be two multi-fuzzy soft hypermodules of dimension $k$ over $M$. If $A \cap B=\varnothing$, then $(\widetilde{f}, A) \widetilde{\sqcup}(\widetilde{g}, B)$ is a multi-fuzzy soft hypermodule of dimension $k$ over $M$.

Proof. According to Definition 13 , we can write $(\widetilde{f}, A) \widetilde{\sqcup}(\widetilde{g}, B)=(\widetilde{h}, C)$, where $C=A \cup B$. As $A \cap B=\varnothing$, it follows that either $c \in A-B$ or $c \in B-A$ for all $c \in C$. If $c \in A-B$, then $\widetilde{h}(c)=M M_{\widetilde{h}(c)}=M M_{\widetilde{f}(c)}=\widetilde{f}(c)$ is a multi fuzzy subhypermodule of $M$ and if $c \in B-A$, then $\widetilde{h}(c)=M M_{\widetilde{h}(c)}=M M_{\widetilde{g}(c)}=\widetilde{g}(c)$ are multi fuzzy subhypermodule of $M$. Therefore, $(\widetilde{f}, A) \widetilde{\sqcup}(\widetilde{g}, B)$ is a multi-fuzzy soft hypermodule of dimension $k$ over $M$.

Theorem 4. Let $(\widetilde{f}, A)$ and $(\widetilde{g}, B)$ be two multi-fuzzy soft hypermodule of dimension $k$ over $M$. Then $(\widetilde{f}, A) \widetilde{\wedge}(\widetilde{g}, B)$ is a multi-fuzzy soft hypermodule of dimension $k$ over $M$.

Proof. According to Definition $12(1)$, let $(\widetilde{f} A) \widetilde{\wedge}(\widetilde{g}, B)=(\widetilde{h}, A \times B)$. We know that $\widetilde{f}(a)=$ $M M_{\widetilde{f}(a)}$, for all $a \in A$, and $\widetilde{g}(b)=M M_{\widetilde{g}(b)}$, for all $b \in B$, is a multi-fuzzy subhypermodule of $M$ and so is $\widetilde{h}(a, b)=M M_{\widetilde{h}(a, b)}=M M_{\widetilde{f}(a) \sqcap \widetilde{g}(b)}$ for all $(a, b) \in A \times B$, because intersection of two multi-fuzzy subhypermodule is also a multi-fuzzy subhypermodule. Therefore, $(\widetilde{f}, A) \widetilde{\wedge}(\widetilde{g}, B)$ is a multi-fuzzy soft hypermodule of dimension $k$ over $M$.

From Theorems 3 and 4, we obtain the following corollary.

Corollary 2. Let $\left\{\left(\widetilde{f}_{i}, A_{i}\right) \mid i \in I\right\}$ be a family of multi-fuzzy soft hypermodule of dimension $k$ over $M$.

(1) If $A_{i} \cap A_{j}=\varnothing$ for all $i, j \in I$ and $i \neq j$, then $\sqcup_{i \in I}\left(\widetilde{f}_{i}, A_{i}\right)$ is a multi-fuzzy soft hypermodule of dimension $k$ over $M$.

(2) $\widetilde{\wedge}_{i \in I}\left(\widetilde{f}_{i}, A_{i}\right)$ is a multi-fuzzy soft hypermodule of dimension $k$ over $M$.

Theorem 5. Let $(\widetilde{f}, A)$ and $(\widetilde{g}, B)$ be two multi-fuzzy soft hypermodules of dimension $k$ over $M$. If $(\widetilde{f}, A) \sqsubset(\widetilde{g}, B)$ or $(\widetilde{g}, B) \sqsubset(\widetilde{f}, A)$, then $(\widetilde{f}, A) \widetilde{V}(\widetilde{g}, B)$ is a multi-fuzzy soft hypermodule of dimension $k$ over $M$.

Proof. Suppose that $(\widetilde{f}, A)$ and $(\widetilde{g}, B)$ be two multi-fuzzy soft hypermodules of dimension $k$ over $M$. By the Definition $12(2)$, we can write $(\widetilde{f}, A) \widetilde{V}(\widetilde{g}, B)=(\widetilde{h}, C)$, where $C=A \times B$, and $\widetilde{h}(a, b)=\widetilde{f}(a) \sqcup \widetilde{g}(b)$ for all $(a, b) \in C$. As $(\widetilde{f}, A)$ and $(\widetilde{g}, B)$ are multi-fuzzy soft hypermodule of dimension $k$ over $M$, we deduce that $\widetilde{f}(a)=M M_{\widetilde{f}(a)}$, for all $a \in A$, and $\widetilde{g}(b)=M M_{\widetilde{g}(b)}$, for all $b \in B$, are multi-fuzzy subhypermodule of $M$. By assumption, $\widetilde{h}(a, b)=\widetilde{f}(a) \sqcup \widetilde{g}(b)$ is a multi-fuzzy soft hypermodule of dimension $k$ over $M$ for all $(a, b) \in C$. Therefore, the $\vee$-union $(\widetilde{f}, A) \widetilde{V}(\widetilde{g}, B)$ a multi-fuzzy soft hypermodule of dimension $k$ over $M$. 
Definition 16. Let $(\widetilde{f}, A)$ and $(\widetilde{g}, B)$ be two multi-fuzzy soft sets of dimension $k$ over $M$. Then, their sum is a multi-fuzzy soft sets $(\widetilde{h}, C)$, where $C=A \cup B$, denoted by $(\widetilde{f}, A) \oplus(\widetilde{g}, B)$ and for all $c \in C$,

$$
\widetilde{h}(c)= \begin{cases}\widetilde{f}(c) & \text { if } \quad c \in A \backslash B \\ \widetilde{g}(c) & \text { if } \quad c \in B \backslash A \\ \widetilde{f}(c) \oplus \widetilde{g}(c) & \text { if } \quad c \in A \cap B,\end{cases}
$$

and for every $z \in M$,

$$
(\widetilde{f}(c) \oplus \widetilde{g}(c))(z)=\bigvee\left\{M M_{\widetilde{f}(c)}(x) \wedge M M_{\widetilde{g}(c)}(y), x, y \in M, z \in x+y\right\}
$$

Theorem 6. Let $(\widetilde{f}, A)$ and $(\widetilde{g}, B)$ be two multi-fuzzy soft hypermodules of dimension k over $M$. Then, $(\widetilde{f}, A) \oplus(\widetilde{g}, B)$ is a multi-fuzzy soft hypermodule of dimension $k$ over $M$.

Proof. It is straightforward.

Definition 17. Let $(\widetilde{f}, A)$ be a multi-fuzzy soft set of dimension $k$ over $M$. The soft set $(\widetilde{f}, A)_{t}=$ $\left\{\left(M_{\widetilde{f}(a)}\right)_{t} \mid a \in A\right\}$, where

$$
\left(M M_{\widetilde{f}(a)}\right)_{t}=\left\{x \in M \mid M M_{\widetilde{f}(a)}(x) \geq t\right\},
$$

for all $t=\left(t_{1}, t_{2}, \ldots, t_{k}\right), t_{i} \in(0,1] 1 \leq i \leq k$, is said to be a $t$-level soft set of the multi-fuzzy soft set $(\widetilde{f}, A)$, where $\left(M M_{\widetilde{f}(a)}\right)$ is a t-level subset of the multi-fuzzy set $M M_{\widetilde{f}(a)}$.

Level set also known as t-cut is a soft set for a multi-fuzzy soft set consisting of all members of universe of discourse have membership value greater than or equal to $t$. The $t$-level cuts are a very useful tool to study the theory of multi-fuzzy soft sets and their applications. It convert the whole multi-fuzzy soft system to soft system (i.e., classical soft set theory system).

This following theorem explores the relation between multi-fuzzy soft hypermodule and $t$-level soft sets.

Theorem 7. Let $(\widetilde{f}, A)$ be a multi-fuzzy soft set of dimension $k$ over $M$. Then, $(\widetilde{f}, A)$ is a multifuzzy soft hypermodule of dimension $k$ over $M$ if and only if for all $a \in A$ and for all $t \in(0,1]$ with $\left(M M_{\widetilde{f}(a)}\right)_{t} \neq \varnothing$ the $t$-level soft set $(\widetilde{f}, A)_{t}$ is a soft hypermodule over $M$.

Proof. Suppose $(\widetilde{f}, A)$ be a multi-fuzzy soft hypermodule of dimension $k$ over $M$. Then, for each $a \in A, M M_{\tilde{f}(a)}$ is a multi-fuzzy subhypermodule of $M$. Suppose that $t \in(0,1]$ with $\left(M M_{\tilde{f}(a)}\right)_{t} \neq \varnothing$ and $x, y \in\left(M M_{\tilde{f}(a)}\right)_{t}$. Then, $M M_{\tilde{f}(a)}(x) \geq t, M M_{\tilde{f}(a)}(y) \geq t$. Thus,

$$
t \leq \min \left\{M M_{\widetilde{f}(a)}(x), M M_{\widetilde{f}(a)}(y)\right\} \leq \inf _{z \in x+y}\left\{M M_{\widetilde{f}(a)}(z)\right\} .
$$

which implies that $M M_{\widetilde{f}(a)}(z) \geq t$ for all $z \in x+y$. Therefore, $x+y \subseteq\left(M_{\widetilde{f}(a)}\right)_{t}$. Moreover, for $x \in\left(M M_{\tilde{f}(a)}\right)_{t^{\prime}}$, we have $M M_{\widetilde{f}(a)}(-x) \geq M M_{\tilde{f}(a)}(x) \geq t$ and $M M_{\tilde{f}(a)}(r x) \geq$ $t$ for all $r \in R$. It follows that $-x, r x \in\left(M M_{\widetilde{f}(a)}\right)_{t}$ for all $r \in R$, We obtain that $\left(M M_{\widetilde{f}(a)}\right)_{t}$ is a sub-hypermodule of $M$ for all $a \in A$. Consequently, $(\widetilde{f}, A)_{t}$ is a soft hypermodule over $M$. Conversely, let $(\widetilde{f}, A)_{t}$ be a soft hypermodule over $M$ for all $t \in(0,1]$. Let $t_{0}=\min \left\{M M_{\tilde{f}(a)}(x), M M_{\widetilde{f}(a)}(y)\right\}$ for some $x, y \in M$. Then, obviously $x, y \in\left(M M_{\widetilde{f}(a)}\right)_{t_{0}}{ }^{\prime}$ consequently, $x+y \subseteq\left(M M_{\tilde{f}(a)}\right)_{t_{0}}$. Thus,

$$
\min \left\{M M_{\widetilde{f}(a)}(x), M M_{\widetilde{f}(a)}(y)\right\}=t_{0} \leq \inf _{z \in x+y}\left\{M M_{\widetilde{f}(a)}(z)\right\} .
$$


Now, $t_{0}=M M_{\widetilde{f}(a)}(x)$ for some $x \in M$. As, by the assumption, every non-empty $t$-level soft set $(\widetilde{f}, A)_{t}$ is a soft hypermodule over $M,-x, r . x \in\left(M M_{\widetilde{f}(a)}\right)_{t_{0}}$ for all $r \in R$. Then, $M M_{\tilde{f}(a)}(-x) \geq t_{0}=M M_{\tilde{f}(a)}(x)$ and $M M_{\tilde{f}(a)}(r x) \geq t_{0}=M M_{\tilde{f}(a)}(x)$. As a result, we get that $M M_{\tilde{f}(a)}$ is a multi-fuzzy subhypermodule of $M$ for all $a \in A$. Consequently, $(\widetilde{f}, A)$ is a multi-fuzzy soft hypermodule of dimension $k$ over $M$.

Definition 18. Let $(\widetilde{f}, A)$ be a multi-fuzzy soft set of dimension $k$ over $M$. We define the soft set $(\widetilde{f}, A)_{0}=\left\{\left(M M_{\widetilde{f}(a)}\right)_{0} \mid a \in A\right\}$, where

$$
\left(M M_{\widetilde{f}(a)}\right)_{0}=\left\{x \in M \mid M M_{\tilde{f}(a)}(x)=M M_{\widetilde{f}(a)}(0)\right\} .
$$

Theorem 8. Let $(\widetilde{f}, A)$ be a multi-fuzzy soft hypermodule of dimension $k$ over $M$. Then, the soft set $(\widetilde{f}, A)_{0}$ is a soft hypermodule over $M$.

Proof. Let $(\widetilde{f}, A)$ be a multi-fuzzy soft hypermodule of dimension $k$ over $M$. Then for each $a \in A, M M_{\widetilde{f}(a)}$ is a multi-fuzzy sub-hypermodule of $M$. Suppose that $x, y \in\left(M M_{\widetilde{f}(a)}\right)$. Then, $M M_{\widetilde{f}(a)}(x)=M M_{\widetilde{f}(a)}(y)=M M_{\widetilde{f}(a)}(0)$. Thus,

$$
M M_{\widetilde{f}(a)}(0)=\min \left\{M M_{\widetilde{f}(a)}(x), M M_{\widetilde{f}(a)}(y)\right\} \leq \inf _{z \in x+y}\left\{M M_{\widetilde{f}(a)}(z)\right\} .
$$

and $M M_{\widetilde{f}(a)}(0) \geq \inf _{z \in x+y}\left\{M M_{\widetilde{f}(a)}(z)\right\}$ which implies that $M M_{\widetilde{f}(a)}(z)=M M_{\widetilde{f}(a)}(0)$ for all $z \in x+y$. Therefore, $x+y \subseteq\left(M M_{\widetilde{f}(a)}\right)_{0}$. Moreover, for $x \in\left(M M_{\widetilde{f}(a)}\right)_{0}$, we have by Proposition 1, $M M_{\widetilde{f}(a)}(-x)=M M_{\widetilde{f}(a)}(x)=M M_{\widetilde{f}(a)}(0)$. and similarly $M M_{\widetilde{f}(a)}(r x)=$ $M M_{\widetilde{f}(a)}(0)$ for all $r \in R$. It follows $-x, r x \in\left(M M_{\tilde{f}(a)}\right)_{0}$ for all $r \in R$, We obtain that $\left(M M_{\widetilde{f}(a)}\right)_{0}$ is a sub-hypermodule of $M$ for all $a \in A$. Consequently, $(\widetilde{f}, A)_{0}$ is a soft hypermodule over $M$.

\section{Image and Pre-Image of Multi-Fuzzy Soft Hypermodules}

In this section, We define the images and pre-images of multi-fuzzy soft hypermodules using Zadeh image and primage operators. Then, we study and investigate their properties. First we begin with the following definition. Suppose that $M^{k} F_{S}^{S}(M, E)=\{(\widetilde{f}, A) \mid A \subseteq E,(f, A)$ is a multi-fuzzy soft set of dimension k over $\mathrm{M}\}$.

Then, $M^{k} F_{S}^{S}(M, E)$ is called a multi-fuzzy soft set class over $M$.

Definition 19. Let $M^{k} F_{S}^{S}\left(M_{1}, E_{1}\right), M^{k} F_{S}^{S}\left(M_{2}, E_{2}\right)$ and $\varphi: M_{1} \rightarrow M_{2}$ and $\psi: E_{1} \rightarrow E_{2}$ be two functions. Then, the pair $(\varphi, \psi)$ is said to be a multi-fuzzy soft function from $M_{1}$ to $M_{2}$.

Definition 20. Let $(\widetilde{f}, A) \in M^{k} F_{S}^{S}\left(M_{1}, E_{1}\right),(\widetilde{g}, B) \in M^{k} F_{S}^{S}\left(M_{2}, E_{2}\right)$ and $(\varphi, \psi)$ be a multifuzzy soft function from $M_{1}$ to $M_{2}$.

(1) The image of $(\widetilde{f}, A)$ under the multi-fuzzy soft function $(\varphi, \psi)$, denoted by $(\varphi, \psi)(\widetilde{f}, A)$, is the multi-fuzzy soft set $(\varphi(\widetilde{f}), \psi(A)) \in M^{k} F_{S}^{S}\left(M_{2}, E_{2}\right)$ such that the multi-fuzzy set $\varphi(\widetilde{f})(t)$ for any $t \in \psi(A)$ is expressed as follows:

$$
M M_{\varphi(\tilde{f})(t)}(y)= \begin{cases}\bigvee_{\varphi(x)=y \psi(a)=t} \bigvee M M_{\tilde{f}(a)}(x) & \text { if } \quad \exists x \in \varphi^{-1}(y) \\ 0, & \text { otherwise }\end{cases}
$$

for all $y \in M_{2}$. 
(2) The pre-image of $(\widetilde{g}, B)$ under the multi-fuzzy soft function $(\varphi, \psi)$, denoted by $(\varphi, \psi)^{-1}(\widetilde{g}, B)$, is the multi-fuzzy soft set $\left(\varphi^{-1}(\widetilde{g}), \psi^{-1}(B)\right) \in M^{k} F_{S}^{S}\left(M_{1}, E_{1}\right)$ such that the multi-fuzzy set $\varphi^{-1}(\widetilde{g})(a)$ is expressed as follows:

$$
M M_{\varphi^{-1}(\widetilde{g})(a)}(x)=M M_{\widetilde{g}(\psi(a))}(\varphi(x))
$$

for all $a \in \psi^{-1}(B)$ and $x \in M_{1}$.

If $\varphi$ and $\psi$ are injective (surjective), then $(\varphi, \psi)$ is said to be injective (surjective).

Furthermore, we define a multi-fuzzy soft homomorphism and show that the homomorphic image and pre-image of a multi fuzzy soft hypermodule are also multi-fuzzy soft hypermodules.

Definition 21. Let $M_{1}, M_{2}$ be two hypermodules and $(\varphi, \psi)$ be a multi-fuzzy soft function from $M_{1}$ to $M_{2}$. If $\varphi$ is a strong homomorphism of hypermodules, then the pair $(\varphi, \psi)$ is called multifuzzy soft homomorphism. If $\varphi$ is a isomorphism and $\psi$ is a one-to-one mapping, then $(\varphi, \psi)$ is said to be multi-fuzzy soft isomorphism.

Theorem 9. Let $M_{1}, M_{2}$ be two hypermodules and $(\varphi, \psi)$ be a multi-fuzzy soft homomorphism from $M_{1}$ to $M_{2}$. If $(\widetilde{f}, A) \in M^{k} F_{S}^{S}\left(M_{1}, E_{1}\right)$ is a multi-fuzzy soft hypermodule, then $(\varphi, \psi)(\widetilde{f}, A) \in$ $M^{k} F_{S}^{S}\left(M_{2}, E_{2}\right)$ is a multi-fuzzy soft hypermodule.

Proof. Let $k \in \psi(A), u, v \in M_{2}$. If $\varphi^{-1}(u)=\varnothing$ or $\varphi^{-1}(v)=\varnothing$, the proof is straightforward. Assume that there exist $x, y \in M_{1}$, such that $\varphi(x)=u$ and $\varphi(y)=v$. Since $(\widetilde{f}, A) \in$ $M^{k} F_{S}^{S}\left(M_{1}, E_{1}\right)$ is a multi-fuzzy soft hypermodule, it follows that for each $a \in A$

$$
\min \left\{M M_{\widetilde{f}(a)}(x), M M_{\widetilde{f}(a)}(y)\right\} \leq M M_{\widetilde{f}(a)}(z)
$$

for all $z \in x+y$. Let $z^{*} \in u+v=\varphi(x+y)$. We obtain $z^{*}=\varphi(z)$. Then, we have

$$
\min \left\{\bigvee_{\varphi(x)=u} M M_{\widetilde{f}(a)}(x), \bigvee_{\varphi(y)=v} M M_{\widetilde{f}(a)}(y)\right\} \leq \bigvee_{\varphi(x)=u} \bigvee_{\varphi(y)=v} M M_{\widetilde{f}(a)}(z) .
$$

Therefore,

$$
\begin{aligned}
\min \left\{M M_{\varphi(\tilde{f})(t)}(u), M M_{\varphi(\widetilde{f})(t)}(v)\right\} & \leq \bigvee_{\psi(a)=t} \bigvee_{\varphi(x)=u} \bigvee_{\varphi(y)=v} M M_{\tilde{f}(a)}(z) \\
& =\bigvee_{\psi(a)=t} \bigvee_{\varphi(z)=z^{*}} M M_{\varphi(\widetilde{f})(t)}(z)
\end{aligned}
$$

for all $z^{*} \in u+v$. Then, we have

$$
\inf _{z^{*} \in u+v}\left\{M M_{\varphi(\widetilde{f})(t)}\left(z^{*}\right)\right\} \geq \min \left\{M M_{\varphi(\widetilde{f})(t)}(u), M M_{\varphi(\widetilde{f})(t)}(v)\right\}
$$

Moreover, for all $r \in R, u \in M_{2}$ where $\varphi(x)=u$ and $x \in M_{1}$, we have

$$
\begin{aligned}
M M_{\varphi(\tilde{f})(t)}(-u) & =\bigvee_{\varphi(-x)=-u \psi(a)=k} \bigvee M M_{\widetilde{f}(a)}(-x) \\
& \geq \bigvee_{\varphi(x)=u \psi(a)=t} \bigvee_{M_{\tilde{f}(a)}}(x) \\
& =M M_{\varphi(\widetilde{f})(t)}(u) .
\end{aligned}
$$

Similarly, $M M_{\varphi(\widetilde{f})(t)}(r u) \geq M M_{\varphi(\widetilde{f})(t)}(u)$. Consequently, $(\varphi, \psi)(\widetilde{f}, A) \in M^{k} F_{S}^{S}\left(M_{2}, E_{2}\right)$ is a multi-fuzzy soft hypermodule. 
Theorem 10. Let $M_{1}, M_{2}$ be two hypermodules and $(\varphi, \psi)$ be a multi-fuzzy soft homomorphism from $M_{1}$ to $M_{2}$. If $(\widetilde{g}, B) \in M^{k} F_{S}^{S}\left(M_{2}, E_{2}\right)$ is a multi-fuzzy soft hypermodule, then $\left(\varphi^{-1}(\widetilde{g}), \psi^{-1}(B)\right) \in M^{k} F_{S}^{S}\left(M_{1}, E_{1}\right)$ is a multi-fuzzy soft hypermodule.

Proof. Let $a \in \psi^{-1}(B), x, y \in M_{1}$. For all $z \in x+y$, we have

$$
\begin{aligned}
\inf _{z \in x+y}\left\{M M_{\varphi^{-1}(\widetilde{g})(a)}(z)\right\} & =\inf _{z \in x+y}\left\{M M_{\widetilde{g}(\psi(a))}(\varphi(z))\right\} \\
& \geq \min \left\{M M_{\widetilde{g}(\psi(a))}(\varphi(x)), M M_{\widetilde{g}(\psi(a))}(\varphi(y))\right\} \\
& =\min \left\{M M_{\left(\varphi^{-1} \widetilde{g}\right)(a)}(x), M M_{\left(\varphi^{-1} \widetilde{g}\right)(a)}(y) .\right\}
\end{aligned}
$$

Similarly for all $r \in R$, we have $M M_{\left(\varphi^{-1} \widetilde{g}\right)(a)}(-x) \geq M M_{\left(\varphi^{-1} \tilde{g}\right)(a)}(x)$ and $M M_{\left(\varphi^{-1} \widetilde{g}\right)(a)}(r x) \geq M M_{\left(\varphi^{-1} \widetilde{g}\right)(a)}(x)$. Therefore, $\left(\varphi^{-1}(\widetilde{g}), \psi^{-1}(B)\right) \in M^{k} F_{S}^{S}\left(M_{1}, E_{1}\right)$ multifuzzy soft hypermodule.

\section{Conclusions}

In this paper, we investigated the formal relationships among notions arising from the theories of algebraic hyperstructures, soft sets, and fuzzy sets. We introduced the concept of multi-fuzzy soft hypermodules over a hypermodule and studied some of their related properties. In our analysis, we include extended theories that introduce fuzziness and softness. In our study, if $k=1$, then the multi-fuzzy soft hypermodule becomes a fuzzy soft hypermodule. Furthermore, we discussed image and pre-image of a multi-fuzzy soft set under the multi fuzzy soft function, and investigated soft homomorphism between multi-fuzzy soft hypermodules.

As algebraic hyperstructures can be used in the modeling of biological phenomena [37], this work can be extended to study the applications of multi-fuzzy soft sets in inheritance phenomena with the base of other algebraic hyperstructures like hypergroups, polygroups, and hyperrings.

Author Contributions: Conceptualization, O.K. and B.D.; methodology, O.K., S.H.-M. and B.D.; formal analysis, O.K.; investigation, O.K.; resources, O.K., S.H.-M. and B.D.; writing-original draft preparation, O.K.; writing—review and editing, O.K., S.H.-M. and B.D.; supervision, B.D.; project administration, S.H.-M.; funding acquisition, S.H.-M. All authors have read and agreed to the published version of the manuscript.

Funding: The research was supported by VAROPS, granted by the Ministry of Defence of the Czech Republic.

Institutional Review Board Statement: Not applicable.

Informed Consent Statement: Not applicable.

Data Availability Statement: Not applicable.

Acknowledgments: The authors are highly grateful to the referees for their constructive suggestions for improving the paper.

Conflicts of Interest: The authors declare no conflict of interest.

\section{References}

1. Marty, F. Sur une generalization de la notion de group. In Proceedings of the 8th Congress Math, Scandenaves, Stockholm, 1934; H. Ohlssons Boktryckeri: Lund, Sweden, 1935; pp. 45-49.

2. Corsini, P.; Leoreanu-Fotea, V. Applications of Hyperstructures Theory; Advanced in Mathematics; Kluwer: Dordrecht, The Netherlands; Springer: Berlin/Heidelberg, Germany, 2003. [CrossRef]

3. Vougiouklis, T. Hyperstructures and Their Representations; Hadronic Press: Palm Harbor, FL, USA, 1999.

4. Davvaz, B.; Leoreanu-Fotea, V. Hyperring Theory and Applications; Hadronic Press, Inc.: Palm Harber, FL, USA, 2007; Volume 115.

5. Davvaz, B.; Cristea, I. Fuzzy Algebraic Hyperstructures; Studies in Fuzziness and Soft Computing; Springer International Publishing: Cham, Switzerland, 2015; Volume 321.

6. Zadeh, L.A. Fuzzy sets. Inf. Control 1965, 8, 338-353. [CrossRef] 
7. Zahedi, M.M.; Bolurian, M.; Hasankhani, A. On polygroups and fuzzy subpolygroups. J. Fuzzy Math. 1995, 3, 1-15.

8. Davvaz, B. Fuzzy $H_{v}$-groups. Fuzzy Sets Syst. 1999, 101, 191-195. [CrossRef]

9. Davvaz, B. Fuzzy $H_{v}$-submodules. Fuzzy Sets Syst. 2001, 117, 477-484. [CrossRef]

10. Rosenfeld, A. Fuzzy groups. J. Math. Anal. Appl. 1971, 35, 512-517. [CrossRef]

11. Zhan, J.M.; Davvaz, B.; Shum, K.P. A new view on fuzzy hypermodules. Acta Math. Sin. 2007, 23, $1345-1356$.

12. Leoreanu-Fotea, V. Fuzzy hypermodules. Comput. Math. Appl. 2009, 57, 466-475. [CrossRef]

13. Massouros, C.G. Free and cyclic hypermodules. Ann. Mat. Pura Appl. IV Ser. 1998, 150, 153-166. [CrossRef]

14. Ameri, R. On categories of hypergroups and hypermodules. J. Discrete Math. Sci. Cryptogr. 2003, 6, 121-132. [CrossRef]

15. Anvariyeh, S.M.; Davvaz, B. Strongly transitive geometric spaces associated to hypermodules. J. Algebra 2009, 322, 1340-1359. [CrossRef]

16. Anvariyeh S.M.; Davvaz, B. On the heart of Hypermodules. Math. Scand. 2010, 106, 39-49. [CrossRef]

17. Norouzi, M.; Leoreanu-Fotea, V. Isomorphism theorems of fuzzy hypermodules. An. St. Univ. Ovidius Constanta 2017, 25, 145-161. [CrossRef]

18. Norouzi, M.; Leoreanu-Fotea, V. Constructions of Quotient of Fuzzy Hypermodules. J. Mult. Valued Log. Soft Comput. 2018, 30, 449-464.

19. Kazanc1, O.; Yamak, S.; Davvaz, B. The lower and upper approximations in a quotient hypermodule with respect to fuzzy sets. Inf. Sci. 2008, 178, 2349-2359. [CrossRef]

20. Molodtsov, D. Soft set theory first results. Comput. Math. Appl. 1999, 37, 19-31. [CrossRef]

21. Maji, P.K.; Biswas, R.; Roy, A.R. Soft set theory. Comput. Math. Appl. 2003, 45, 555-562. [CrossRef]

22. Maji, P.K.; Biswas, R.; Roy, A.R. Fuzzy soft sets. J. Fuzzy Math. 2001, 9, 589-602.

23. Aktaş, H.; Çağman, N. Soft sets and soft groups. Inf. Sci. 2007, 177, 2726-2735. [CrossRef]

24. Aygunoğlu, A.; Aygun, H. Introduction to fuzzy soft groups. Comput. Math. Appl. 2009, 58, 1279-1286. [CrossRef]

25. Leoreanu-Fotea, V.; Feng, F.; Zhan, J. Fuzzy soft hypergroup. Int. J. Comput. Math. 2012, 89, 963-974. [CrossRef]

26. Ameri, R.; Norouzi, M.; Hedayati, H. Application of fuzzy sets and fuzzy soft sets in hypermodules. Rev. Real Acad. Cienc. Exactas Fis. Nat. Ser. Mat. 2013, 107, 327-338. [CrossRef]

27. Zhan, J.M.; Cristea, I. Characterizations of fuzzy soft Gamma-hemirings. J. Intell Fuzzy Syst. 2014, 26, 901-911. [CrossRef]

28. Sebastian, S.; Ramakrishnan, T.V. Multi-fuzzy sets: An extension of fuzzy sets. Fuzzy Inf. Eng. 2011, 3, 35-43. [CrossRef]

29. Onasanya, B.O.; Hoskova-Mayerova, S. Multi-fuzzy group induced by multisets. Ital. J. Pure Appl. Math. 2019, 41, 597-604.

30. Al-Tahan, M.; Hoskova-Mayerova, S.; Davvaz, B. Fuzzy multi-polygroups. J. Intell Fuzzy Syst. 2019, 38, 2337-2345. [CrossRef]

31. Al Tahan, M.; Hoskova-Mayerova, S.; Davvaz, B. Some Results on (Generalized) Fuzzy Multi- $H_{v}$-ideals of $H_{v}$-Rings. Symmetry 2019, 11, 1376. [CrossRef]

32. Yang, Y.; Tan, X.; Meng, C. The multi-fuzzy soft set and its application in decision making. Appl. Math. Modell. 2013, 37, 4915-4923. [CrossRef]

33. Akın, C. Multi-fuzzy soft groups. Soft Comput. 2021, 25, 137-145. [CrossRef]

34. Krasner, M. A class of hyperring and hyperfields. Int. J. Math. Math. Sci. 1983, 6, 307-312. [CrossRef]

35. Bordbar, H.; Cristea, I.; Novak, M. Height of Hyperideals in Noetherian Krasner Hyperrings. Univ. Politeh. Buchar. Sci. Bull.-Ser. A-Appl. Math. Phys. 2017, 79, 31-42.

36. Bordbar, H.; Cristea, I. Height of prime hyperideals in Krasner hyperrings. Filomat 2017, 31, 6153-6163. [CrossRef]

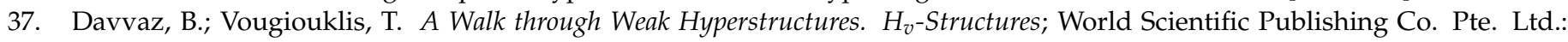
Hackensack, NJ, USA, 2019. 